\title{
ANÁLISIS DE RIESGO DE LAS PRINCIPALES ACCIONES ENLISTADAS EN LA BOLSA DE VALORES DE LIMA
}

\author{
RISK ANALYSIS OF MAJOR ACTIONS LISTED \\ IN THE STOCK EXCHANGE OF LIMA
}

\author{
Nicko Alberto Gomero Gonzales ${ }^{*}$ \\ Ana María Gutiérrez Huby**
}

Docentes Principales de la Facultad de Ciencias Contables

\author{
Universidad Nacional Mayor de San Marcos-UNMSM / Lima-Perú
}

[Recepción: Julio de 2013/ Conformidad: Agosto 2013]

\section{RESUMEN}

Por naturaleza, el mercado bursátil y las acciones que en ella se negocia, se caracterizan por su alto grado de volatilidad o riesgo; es por ello que los inversionistas, es poco común que apuesten por un solo activo, todo lo contrario siempre inclinan sus preferencias a la estructuración de carteras ya que de esta forma, producto de la diversificación podrán lograr puntos óptimos de convergencia entre el riesgo y rendimiento esperado. Existen métodos que permiten determinar la volatilidad de un activo, entre ellos está la varianza y desviación típica. Por otro lado, también se puede utilizar para este fin la Beta, indicador que señala la manera como se mueve las acciones ante cambios del mercado, la cuantificación de estos indicadores en el presente articulo, señalan que en el mercado bursátil se puede encontrar activos con diferentes grados de riesgo.

\section{Palabras Clave:}

Portafolio, mercado bursátil, acciones, Beta de un activo.

\begin{abstract}
By nature, the stock market and the shares that it is negotiated, are characterized by their high degree of volatility or risk, which is why investors, it is uncommon to bet on a single asset, on the contrary always bow their preferences to structuring portfolios because this way, product diversification may achieve optimal points of convergence between risk and expected return. There are methods for determining the volatility of an asset, among them is the variance and standard deviation on the other hand can also be used for this purpose the Beta indicator pointing the way to change the actions of market moves, the quantification of these indicators in this article indicate that the stock market can find assets with different degrees of risk
\end{abstract}

\section{Keywords:}

Portfolio, stock market, stocks, Beta of an archive.

\footnotetext{
* Doctor en Ciencias Económicas. Magíster en Contabilidad con mención en Banca y Finanzas-UNMSM.

Email: nickgo_6@msn.com,gomero_econ@yahoo.es.

** Doctora en Administración-USP. Magister en Gestión Empresarial-UNMSM. Email: anamaria01053@yahoo.es
} 


\section{INTRODUCCIÓN}

El Mercado Bursátil Nacional, a pesar de los comportamientos cíclicos y turbulentos que experimenta por la economía mundial, ha venido logrando resultados favorables, tal es el caso, que sus fortalezas lo ubicaron como una de las Bolsas con mayores expectativas de crecimiento de la región. Si bien, es cierto que este mercado es pequeño y las transacciones por día está muy por debajo del promedio regional, pero no deja de ser un importante destino para tomar decisiones de inversión. Como se conoce, algunos agentes económicos entran a este escenario con fines especulativos, pero otros, como los institucionales optarán por utilizarlos como una estratégica fuente de financiamiento a largo plazo. Sin duda, el mercado de deuda y de capitales, se han constituido en importantes apalancadores del crecimiento económico.

La importancia de este mercado no solo se centra en su capacidad de emitir dinero fresco para solventar proyectos de inversión o generar riqueza por operaciones especulativas, sino que además, su relevancia se centra en que su performance se constituye en el punto de referencia para evaluar el comportamiento de la economía y por qué no decir, para ensayar algunas estimaciones macroeconómicas. Como se argumenta, si el mercado bursátil se debilita, se debe a algo "extraño" que está ocurriendo en el escenario económico, financiero y político del país el cual es transmitido en forma inmediata a este marcado.

Este mercado por si es muy sensible, todo evento exógeno rápidamente se reflejará en el comportamiento de los índices y con ello, de las acciones. La volatilidad que se experimente en el rendimiento del mercado y de los activos financieros, para algunos representará oportunidades de ganancia y para otros, desequilibrios en sus cuentas financieras. Como se argumenta, la Bolsa es un mercado de incertidumbre, pero esto no implica que los agentes económicos no puedan tomar posiciones que les conlleve a optimizar sus rentabilidades esperadas. Para ello, existen modelos financieros, como el de Markowitz. Sharpe,
CAMP, ARCH GARCH, el beta, que facilitan al operador bursátil lograr la mejor estructura de portafolio. $\mathrm{O}$ algo más simple, el modelo de covarianzas o correlaciones permitiría adecuar la combinación de cartera que mejor se ajuste al perfil del inversionista.

En el presente artículo, se analiza un conjunto de acciones que se cotizan en la Bolsa de Valores y están enlistadas en el Índice Selectivo, tomando como base el comportamiento de su rentabilidades y debidamente contrastadas con el rendimiento del mercado. En el presente estudio, se llega a determinar cada uno de las betas, indicador que proyecta el grado de sensibilidad del activo con relación al comportamiento del mercado.

\section{VULNERABILIDADES Y VOLATILIDADES DE LOS MERCADOS BURSÁTILES GLOBALES: EVALUACIÓN DEL ENTORNO}

Con las ultimas crisis globales, ha quedado evidenciado que el mercado bursátil ha profundizado su grado de volatilidad, su inestabilidad, es así como lo demuestran las estadísticas financieras, se ha traducido en pérdidas importantes para aquellos inversionistas de carácter particular e institucional que tienen por cultura participar en estos mercados altamente especulativos.

El grado de volatilidad de estos mercados no solo ha generado impactos en los indicadores financieros de los negocios; al contrario, sus efectos han llegado a trastocar cimientos económicos, inclusive de carácter estructural, tal como lo sucedido en Zona del Euro y Norteamérica, países que han experimentado caídas sostenidas de sus principales indicadores económicos y sociales. En este caso, cabe precisar los efectos perniciosos de la crisis bursátil especialmente en España cuya tasa de paro, llegó en promedio al 19\%, superando en exceso a lo alcanzado por los integrantes de la Zona del Euro. Sobre el IBEX35, que es su principal indicador bursátil, se puede afirmar que es una de las más volatilizadas y riesgosa de esa región.

Las cifras manejadas por el $\mathrm{FMI}^{1}$, resaltan la inestabilidad de aquellos países que aún mantienen por

1 FMI. Informe sobre la estabilidad financiera mundial. Abril 2013.

44/ QVIPURAMAYOC | Vol. 21(40) 2013 
ahora la supremacía económica global, nos estamos refiriendo a EE.UU, país que no ha permanecido al margen del impacto de las crisis financieras, tal es así que su PBI real, el cual es simétrico a su deuda externa, no llega a una cifra que sobrepase el $3 \%$, que si bien es cierto, en estos últimos meses ha entrado en una etapa de reacomodo con el aumento en los niveles confianza del consumidor, pero todavía se siguen presentando señales de cierta debilidad en su avance económico y social. La tasa de paro llegó en promedio al $9 \%$, cifra que en escenarios normales debe llegar a la tasa natural ${ }^{2}$, indicador que no debe preocupar a ninguna gestión de gobierno.

Los principales índices bursátiles en EE.UU aun presentan señales de riesgo, los inversionistas especulativos no demuestran su total confianza para apostar sus capitales en estos mercados debido a que se mantienen latentes los temores de una nueva crisis soberana. EINASDAQ, elDOWJONES ${ }^{3}$, entre otros indicadores bursátiles vienen proyectando señales de desconfianza de los operadores económicos. Si bien, estos mercados se caracterizan por su elevada inestabilidad, pero por los grados de incertidumbre que presentan a dejado de ser prioridad en la cartera de inversiones de los megas especuladores, por ello las acciones que están detrás de cada uno estos índices no dan un salto importante en sus cotizaciones.

Acciones de importantes empresas de tipo global como Coca Cola, GM, APPLE, MICROSOFT ${ }^{4}$, entre otros de este mismo nivel, dentro de sus características de fortalezas financieras, aun muestran claros indicios de tendencias decrecientes, resultado que no es más que síntomas de débiles posiciones de los especuladores para con estos activos. Hay que recordar, que la quiebra y salida del segundo Banco más importante de EE.UU, fue producto de la caída sostenida del mercado bursátil norteamericano, que sin duda, fue respuesta a lo sucedido con los créditos hipotecarios, los cuales no tuvieron una adecuada gestión. El resultado obtenido abrió fisuras de carácter estructural motivando la participación de los gestores de las políticas públicas. Estos hechos llegan a demostrar que los impactos bursátiles, pueden debilitar a economías que supuestamente se creían fuertes e inmunes a todo tipo de crisis global.

La crisis de 1929, conocido como el jueves negro fue, una de las inestabilidades mas grandes del mercado del siglo XX, que introdujo a las economías mundiales, especialmente a la de EE.UU a los peores escenarios recesivos. El Crak Bursátil del año mencionado tradujo riquezas financieras en cuentas menesterosas de bancos, y la tasa de desempleo fue denominador común en todas las áreas sociales, este último efecto, también se observó en la crisis del 2008, alcanzando la tasa de paro cifras de dos dígitos y no visto en los últimos 50 años. Los mercados bursátiles por sus alta dosis especulativa, es muy sensible a las perturbaciones económicas y políticas. Hay que precisar que, las deficientes políticas públicas se traducen en mercados bursátiles inestables y de alto riesgo, para demostrar esta afirmación solo basta revisar las cifras alcanzadas por Grecia, país calificado como inviable e insostenible por las Calificadoras de Riesgo.

El FMI y las Agencias Calificadoras de Riesgo siempre están atentos para evaluar las medidas correctivas de los gobiernos y las señales de ingobernabilidades, siendo la primera economía del mundo una de las que experimentó una tasa de castigo a dicha calificación, remeciendo dicho sea de paso a la economía global. Pasar de AAA a una calificación de AA, fue uno de los peores golpes de credibilidad a la gestión gubernamental de ese país.

2 En toda economía, aunque sea la más desarrollada siempre está presente el desempleo llamado natural.

3 Cifras obtenidas en la página electrónica de: www.finanzas.com

4 Cifras obtenidas de: www.finanzas.com 
Si bien, los mercados de renta variables como las acciones presentan características de sensibilidad, existen otros activos financieros como los Derivados ${ }^{5}$ que también fueron las causantes de la crisis global. En ocasiones son instrumentos que son utilizados para lograr coberturas ante las volatilidades de los mercados, pero por otra parte sus transacciones están cubiertas por operaciones especulativas, peor aún, estas actividades se desarrollan sin tener como contrapartidas activos reales que los respalden. Como dicen los expertos, solo se negocian papeles.

\section{MERCADO BURSÁTIL NACIONAL}

El mercado de valores de Perú, si bien es uno de los más pequeños del mundo, también es caracterizado por su comportamiento especulativo y de elevada sensibilidad. Los agentes económicos locales y globales interactúan con operaciones de este tipo, realizando transacciones financieras con documentos de renta variable (acciones) y renta fija (bonos). Su cotización de estos activos va a depender de cuanto sea su oferta y su demanda en un periodo de tiempo. Tal como señalan los especialistas, la Bolsa de Valores es el mejor ejemplo de un mercado libre, pero de alto corte especulativo. Este concepto se ajusta a la definición que ensaya la BVL ${ }^{6}$.

La Bolsa de Valores señala que, "Una empresa se puede financiar mediante la emisión y colocación de distintos tipos de valores. Los valores más comunes son las acciones y los bonos. Cuando una empresa decide obtener recursos mediante una emisión y colocación de valores, debe conocer primero las obligaciones en que incurriría al listar en bolsa, entre los que destacan la entrega puntual de información relevante según la normativa peruana, regulada por $S M V$ ". Asimismo la BVL, específica que "los accionistas de la compañía deben estar dispuestos a compartir el accionariado con personas que no necesa- riamente van a conocer. Estos nuevos accionistas pueden proveer financiamiento y conocimiento. Los accionistas originales deben estar convencidos de que su empresa requiere de estos aportes y de que crearán valor para su negocio."

En los conceptos ensayados por la BVL se puede apreciar que el mercado bursátil aparte de ser una fuente para acumular riqueza a través de operaciones especulativas, también es utilizado para que las empresas y gobiernos puedan captar recursos financieros "frescos" para solventar sus actividades de inversión de largo plazo, así lo evidencian las operaciones del gobierno, al mostrar preferencia para emitir bonos soberanos en el mercado local.

El desempeño de la Bolsa se mide por los resultados que pueda alcanzar el Índice General y Selectivo. Si estos índices presentan signos positivos, es una clara señal que las acciones están obteniendo rentabilidades crecientes, es decir, las condiciones de la economía nacional, traducido en mayores exportaciones e inversiones, complementados con cuentas fiscales equilibradas, estarían creando escenarios favorables para los activos de renta variable y de riesgo. Su volatilidad en este caso le permitirá alcanzar cifras atractivas para realizar operaciones especulativas.

Cabe precisar, que a partir de julio de 1993, se viene calculando el Índice Selectivo de la Bolsa de Valores de Lima (ISBVL), indicador que mide las variaciones de las cotizaciones de las 15 Acciones más representativas de la Bolsa de Valores de Lima. Este índice permite mostrar la tendencia del mercado bursátil en términos de los cambios que se producen en los precios de estos activos. Las empresas enlistadas en el Índice Selectivo son de tipo corporativo y de alta representatividad en el sector productivo, siendo las mineras, las que tienen mayor incidencia en los resultados, tal es así que VOLCAN B posee la más alta ponderación en el resultado del Selectivo,

\footnotetext{
5 Principales Derivados : opciones y futuros de activos reales y financieros, forward. Estos contratos se negocian en el mercado de derivados . La primera regulación de los mercados organizados de futuros y opciones surge en marzo de 1989, con unas características que derivan de dos hechos. El primero es la constitución de importantes carteras de deuda pública, cuyos tenedores demandaban instrumentos adecuados para la cobertura del riesgo de mercado en el que se veían incursos. El segundo son las limitaciones legislativas vigentes en ese momento, básicamente la falta de desarrollo reglamentario de la Ley del Mercado de Valores, que impedía abordar el tema con globalidad, y la prohibición, hasta entonces, de que los clientes pudieran operar a plazo en el mercado secundario de deuda anotada.

6 Ver la página electrónica, //www.bvl.com.pe/emision/brochure.pdf
}

46/ QVIPURAMAYOC | Vol. 21(40) 2013 
siendo esta acción una de las liquidas del mercado bursátil. Este grado de participación de las mineras en la BVL, nos indica su elevado grado de dependencia al performance de la economía internacional, especialmente de las cotizaciones de los commodities en los mercados globales, oscilación que está anclada al desempeño de la economía China, principal socio comercial de Perú en la transacción de materias primas.

\begin{tabular}{|cllc|}
\hline \multicolumn{4}{|c|}{ CARTERA DEL INDICE SELEC TIVO BVL } \\
\hline \multicolumn{4}{|c|}{ (vigente a partir del 2 de ENERO de 2013) } \\
\hline N $^{\circ}$ & Nombre de valor & Nemónico & Peso \% \\
\hline 1 & Volcan "B" & VOLCABC1 & $15.2595 \%$ \\
\hline 2 & Rio Alto Mining & RIO & $9.9863 \%$ \\
\hline 3 & Ferreycorp & FERREYC1 & $8.4557 \%$ \\
\hline 4 & Cerro Verde & CVERDEC1 & $6.7703 \%$ \\
\hline 5 & ADR Buenaventura & BVN & $6.6320 \%$ \\
\hline 6 & Graña y Montero & GRAMONC1 & $6.2668 \%$ \\
\hline 7 & Maple Energy & MPLE & $6.1957 \%$ \\
\hline 8 & Minsur Inv. & MINSURI1 & $5.7155 \%$ \\
\hline 9 & U And de Cementos & UNACEMC1 & $5.5719 \%$ \\
\hline 10 & Relapasa & RELAPAC1 & $5.3980 \%$ \\
\hline 11 & Alicorp & ALICORC1 & $5.3666 \%$ \\
\hline 12 & Credicorp & BAP & $4.8150 \%$ \\
\hline 13 & Intergr Financ Services & IFS & $4.6224 \%$ \\
\hline 14 & Bco. Continental & CONTINC1 & $4.6178 \%$ \\
\hline 15 & Cementos Pacasmayo & CPACASC1 & $4.3263 \%$ \\
\hline
\end{tabular}

Fuente : BVL
Por otro lado, el Índice General de la Bolsa de Valores de Lima (IGBVL) que mide el comportamiento del mercado bursátil, sirve para establecer comparaciones respecto de los rendimientos alcanzados por los diversos sectores (industrial, bancario, agrario, minero, de servicios públicos). Se determina a partir de una cartera formada por los títulos de renta variable significativa de la negociación bursátil, seleccionadas con base en su frecuencia de negociación ${ }^{7}$, monto de negociación y número de operaciones.

Cuando los índices bursátiles, presentan tendencias alcistas, los rendimientos esperados de quienes realizan operaciones de compra y de venta superan a lo que se pueda alcanzar en otros mercados financieros y reales. Las operaciones especulativas bajo este escenario se convierten en los de máxima rentabilidad, caso contrario sucede cuando los indicadores bursátiles se deprimen, situación que evidencia lo sucedido con la última crisis global, donde los inversionistas instituciones perdieron importantes capitales, tal como fue experimentada por las empresas que administran Fondos de Pensiones.

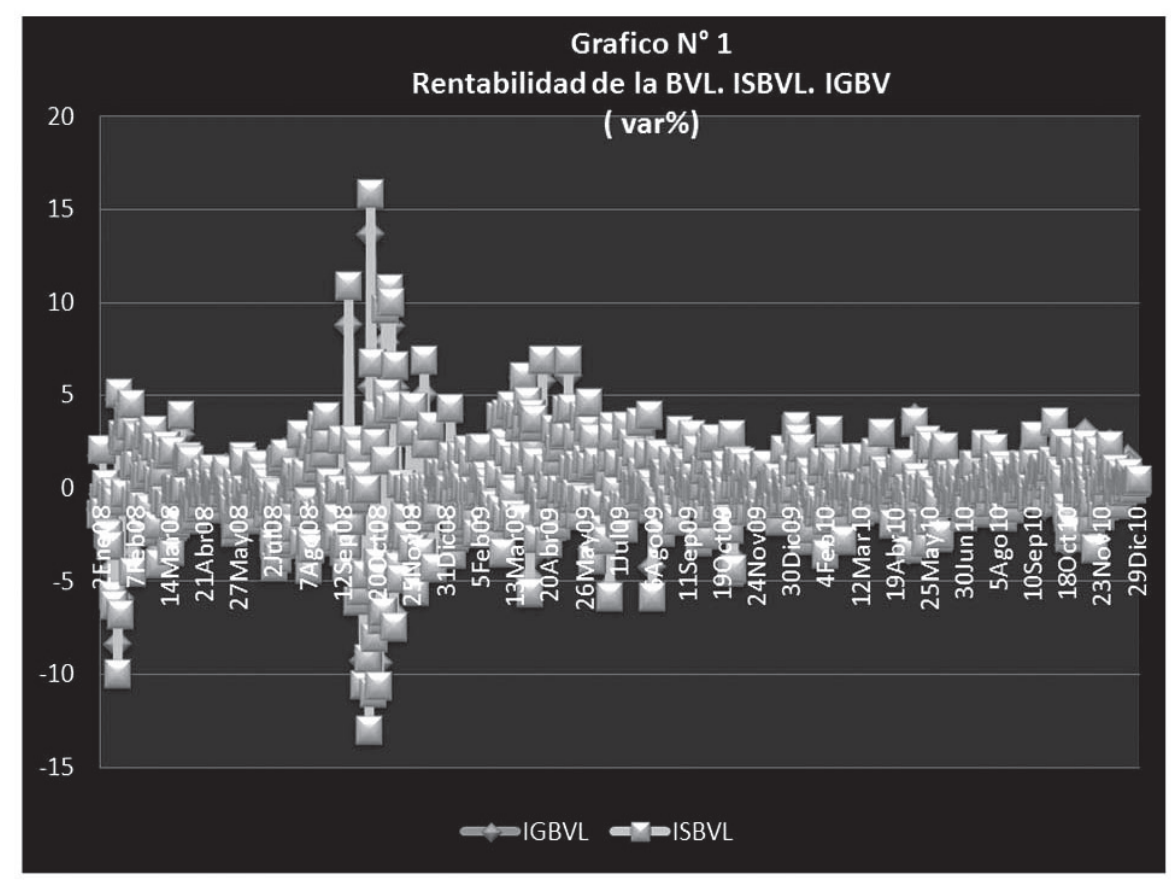

Fuente : BVL

Elaboración propia

7 Cuanto mayor sea la frecuencia de negociación de una acción se podría señalar que es más líquida. Los especuladores tendrían propensión demandar estos activos para abrir y cerrar posiciones en el mercado bursátil. 
Tal como se muestra en el gráfico $\mathrm{N}^{\circ} 1$, las peores caídas de la BVL se experimentaron en el 2008/2009, producto de la crisis mundial, pero esta caída no fue tan fuerte como se observó en las Bolsas Europeas, como lo sucedido con el IBEX de España, que fue una de las que más retrocedió con la crisis global.

\section{EVALUACIÓN DE RIESGO DE LAS ACCIONES QUE SE NEGOCIAN EN LA BVL. EL BETA DEL ACTIVO}

Jeff Madura (2010), señala que "La Beta sirve como una medición del riesgo debido a que se puede usar para medir una distribución de la probabilidad de los rendimientos con base en un conjunto de rendimientos de mercado. La Beta es interesante para aquellos inversionistas interesados en el riesgo sistemático".

El beta indica cuan sensible o riesgoso es la acción. Puede alcanzar los siguientes resultados:

\section{B mayor a 1}

B menor a 1

B igual a 1

En el primer caso, se dice que el activo es de alto riesgo, la variabilidad de su rendimiento supera al del mercado, si este coeficiente es menor a 1, la acción es menos sensible que el mercado y cuando es 1 , el riesgo o rendimiento del activo es simétrico al del mercado.

Un inversionista particular o institucional puede demandar activos de altos o bajos betas, todo depende de cual sea su preferencia al riesgo, o en todo caso puede optar por estructurar portafolios, con la finalidad de diluir los riesgos por la volatilidad propia de estos activos. Cuando los mercados están al alza es recomendable estructurar portafolios de altos betas, que superen a 1 , de esta forma alcanzarán rendimientos muy superiores a los del mercado bursátil. A continuación, se estructura una fórmula que permite obtener betas de cartera $(\mathrm{Bc})$.

$$
\mathrm{Bc}=\mathrm{w} \mathbf{1}^{*} \mathrm{~B} 1+\mathrm{w} 2{ }^{*} \mathrm{~B} 2
$$

$\mathrm{W} 1=$ participación del activo 1 en el portafolio $\mathrm{B} 1=$ beta del activo 1

W2= participación del activo 2 en el portafolio $\mathrm{B} 2=$ beta del activo 2
La combinación de los activos, puede inducir a estructurar carteras diversificadas de diferentes grados de riesgo, gestión de cobertura que tiende a minimizar los riesgos por inversión.

Para valores o acciones, su Coeficiente Beta $(\beta)$ se calcula usando análisis de regresión contra un índice representativo del valor del mercado, por ejemplo el IGBVL, en la Bolsa peruana.

El beta se puede calcular utilizando la siguiente formula:

$$
\mathbf{B}=\frac{\operatorname{COV}(\mathbf{R i}, \mathbf{R m})}{\operatorname{Var}(\mathbf{R m})}
$$

Matemáticamente para determinar el beta se tiene que determinar la covarianza entre el rendimiento del activo y del mercado y dividirlo entre la varianza del rendimiento del mercado, resultado que debe coincidir con la pendiente de una línea de regresión lineal del tipo:

$$
\mathbf{Y}=\boldsymbol{\beta} \mathbf{0}+\boldsymbol{\beta} \mathbf{1}(\mathbf{X})
$$

Donde $: \mathrm{Y}=$ Rendimiento del activo

$\mathrm{X}=$ Rendimiento del mercado

$\mathrm{B} 1=$ Beta del activo

Beta, como argumentan los analistas bursátiles, mide solo el riesgo sistemático, es decir aquel riesgo que no es posible eliminar diversificando la cartera en distintos tipos de activos. De tal forma, que un inversor que tiene su dinero concentrado en pocos negocios no encontrará al beta como una medida representativa de su riesgo; puesto que el mismo subestimará el riesgo específico.

Un inversionista especulativo solo debe centrar su preocupación en el riesgo de mercado, ya que el riesgo único lo tendera a minimizar con una buena gestión de cartera. En este sentido, para minimizar los riesgos es recomendable que tome posiciones cruzando activos que tengan coeficientes de correlaciona negativo $(r=-1)$. Una cartera cuanto más cerca de -1 este su correlación menor será riesgo. El riesgo se elimina con activos cuya correlación sea -1 . una mayor posición de riesgo implica demandar activos cuya correlación sea 1. 


\section{RESULTADOS DE ANÁLISIS DE RIESGO DE EMPRESA ENLISTADAS EN LA ISBVL}

¿Cuáles son los betas de las empresas mineras que cotizan en la BVL?. Para responder esta pregunta cabe precisar que, se ha procesado información histórica de rendimientos de activos de renta variable (acciones) enlistadas en el Indicé Selectivo, en forma diaria y de los últimos 12 meses. En un principio se analizó el precio, para posteriormente cuantificar su volatilidad. Asimismo, se procesó información del Índice Bursátil, específicamente del Selectivo, ya que la determinación de este indicador de volatilidad exige contrastar el performance de los rendimientos de los activos con los resultados del mercado. Se utilizó el método de la Regresión Lineal Simple. Cuyo resultado fue corroborado, cruzándolo con el método de la covarianza y varianza de los rendimientos de los activos y del mercado respectivamente.

Se ha detectado acciones que presentan alta volatilidad, con betas por encima de 1 , lo cual significa que sus rendimientos oscilan más rápidamente que el comportamiento del mercado. Pero también hay activos, como Cemento Pacasmayo que presenta bajo indicador de volatilidad $($ Beta $=0.57)$. Con esta acción, un inversionista debe esperar ganar un $0.57 \%$ por cada punto $\%$ que avance la Bolsa, o en todo caso perder esta misma cantidad si este mercado tiene caídas del $1 \%$. Es decir sus ganancias y pérdidas serían moderadas, por ello se le califica como un activo de bajo riesgo. Cemento Pacasmayo es una empresa ligada a la actividad de la construcción, sus Estados Financieros y el precio de mercado de sus acciones reflejan la coyuntura inmobiliaria del país.

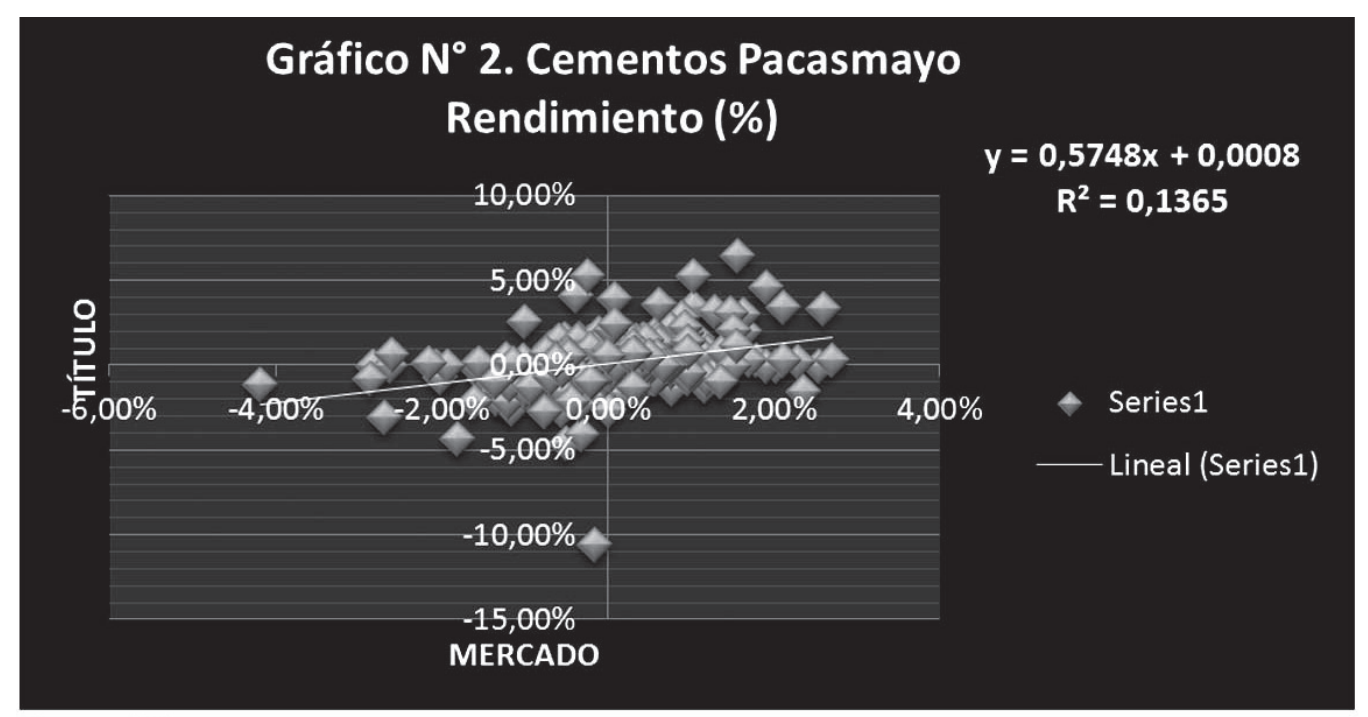

Fuente: BVL

Elaboración propia

Tal como se mencionó, en un principio las acciones mineras, entre ellas Volcán B, como se puede apreciar en el gráfico $\mathrm{N}^{\circ} 3$, su grado de volatilidad llega a 1.31 , señalando que con este activo se puede ganar $0.31 \%$ más por cada punto \% que avanza la BVL, caso contrario se puede obtener pérdidas en esta misma magnitud, por cada $1 \%$ que retrocede el mercado de valores.
Los rendimientos con Volcán B, cuyo beta es mayor a 1, están volatilizados, lo cual podría conllevar importantes ganancias o pérdidas para el inversionista especulativo. Cuando los precios de los minerales, mejoran en el mercado mundial, producto del auge económico de la China, hay que apostar por esta acción, debido a que las ganancias obtenidas serán significativas y estarían por encima del promedio del mercado. 


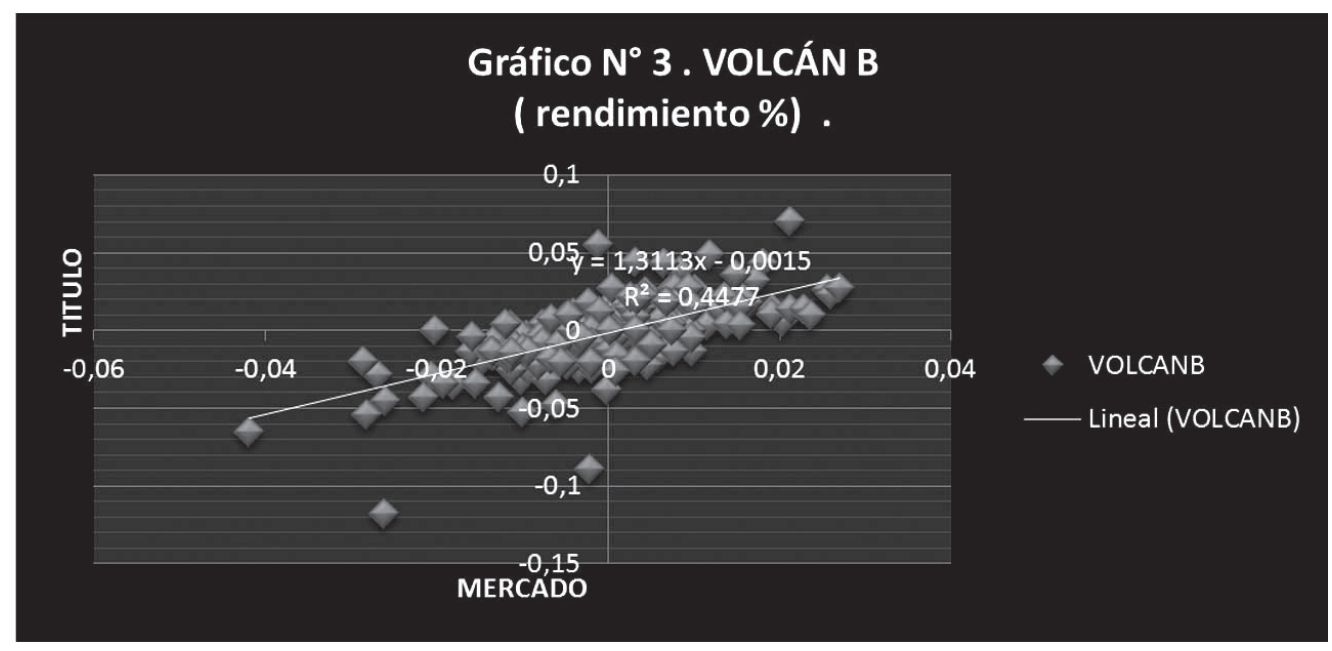

Otra acción minera que presenta elevada volatilidad es Rio Blanco, cuyo beta es de 1.25. Su grado de riesgo de este activo está cerca que de Volcán B. al comparar este activo se tendría que esperar pérdidas o ganancias en un $0.25 \%$ por encima del mercado. Si el mercado de valores presenta tendencias alcistas, un inversionista estaría tomando posición ventajosa con una cartera conformada con estos dos activos, pero si las señales del mercado, inducen a caídas sostenidas, lo recomendable seria cerrar posiciones, debido a que las pérdidas superarían al promedio del mercado. Esta cartera seria de alto riesgo.

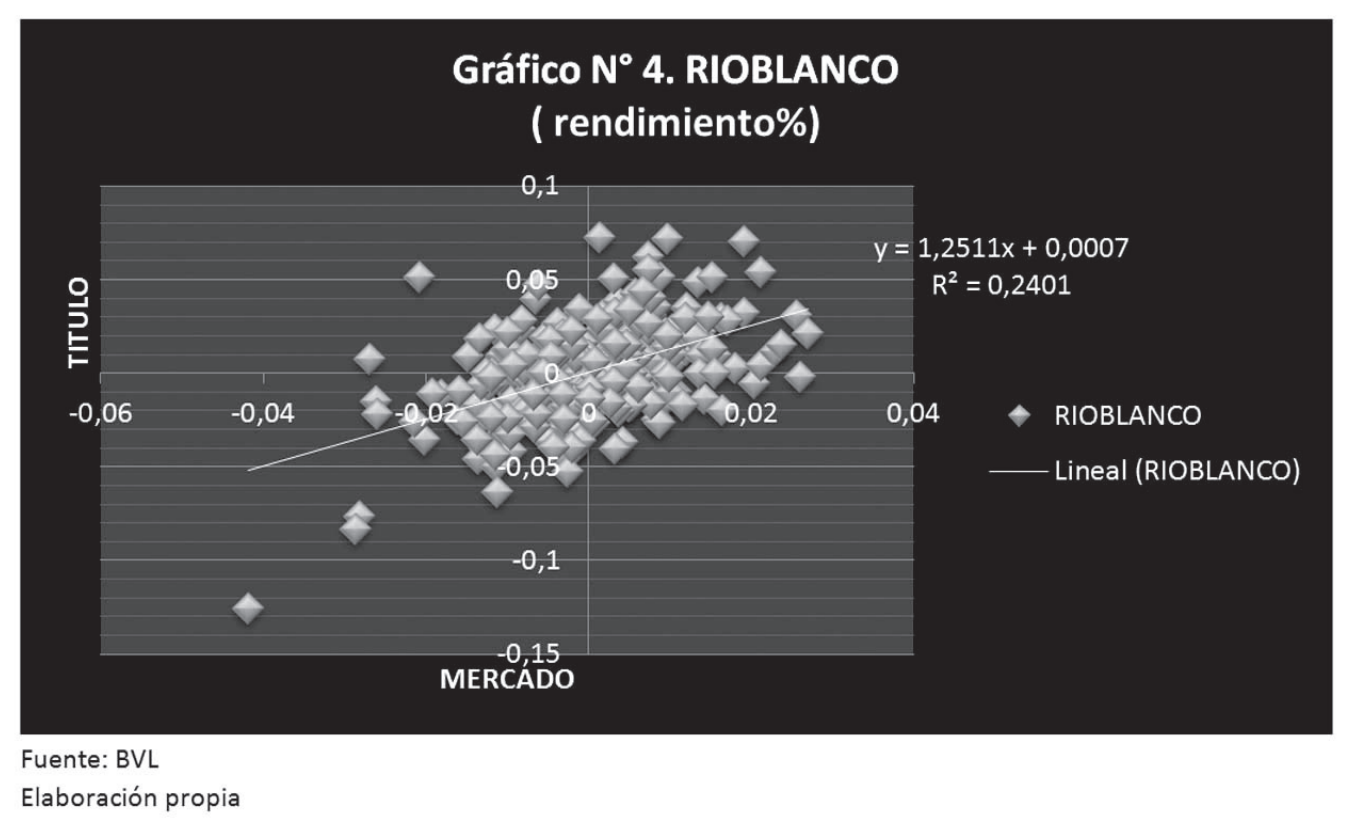

El riesgo de los activos de rentas variables no solo se puede medir utilizando el beta, sino que otros estadísticos válidos para este fin es la desviación típica y el coeficiente de correlación. Estos instrumentos matemáticos serán utilizados para analizar posiciones de cartera con activos enlistados en la BV de Lima y verificar la operatividad de la teoría de Markowitz. Modelo que se desarrollará para informes posteriores. 


\section{CONCLUSIONES}

1. El mercado bursátil global, después de la crisis que originó la caída de instituciones financieras internacionales y desequilibrios de las cuentas gubernamentales de muchos países involucrados en este escenario de turbulencia, aun no logra dar señales de confianza. Es por ello que los inversionistas globales se muestran cautelosos en el momento de estructurar sus portafolios de activos. El NASDAQ, EL IBEX, DOW JONES, entre otros índices bursátiles, no muestran señales claras de recuperación y de crecimiento sostenido.

2. El mercado bursátil nacional, si bien se caracteriza por ser pequeño y poco profundo, en comparación con otras bolsas de la región y del mundo, pero debido a las fortalezas macroeconómicas del país, ha experimentado crecimientos importantes y sostenidos en su rentabilidad, proyectando con ello, buenas señales a los inversionistas locales e internacionales.

3. El hecho que la Bolsa de Valores se caracteriza por su elevada volatilidad, esto no significa que las empresas, el gobierno, inversionistas no institucionales, la consideren como un importante escenario para realizar operaciones especulativas de corto plazo o en todo caso, obtener liquidez para financiar sus proyectos de inversión.

4. El beta es un indicador de volatilidad de los activos de renta variable. Su determinación implica contrastar el rendimiento del activo con el del Mercado de Valores. Bajo esta concepción teórica, las acciones cementeras presentan señales de baja volatilidad, contrariamente, los títulos mineros, son los que proyectan mayores señales de riesgo ya que el coeficiente de volatilidad (beta) es mayor a la unidad. Con este tipo de acciones los inversionistas deberían de esperar ganar más que el mercado bursátil.

\section{REFERENCIAS BIBLIOGRÁFICAS}

1. Michael C. Eugene Bringham (2007). Finanzas Corporativas. Cengaje Learnig Editores.

2. Stephen Ross - Randolph W. Westerfield Bradford D. Jordán (2001) Fianzas Corporativas. Editorial: - McGraw-Hill quinta edición.

3. Richard A. Brealey - Stewart C. Myers (1993) Finanzas Corporativas. Editorial: McGraw-Hill Mexico Edición: Cuarta.

4. Jeff Madura (2010). Mercado e Instituciones Financieras. CENAGE LEARNIG. México. Cuarta edición.

\section{REFERENCIAS ELECTRÓNICAS}

1. www.http://www.bvl.com.pe. Indicadores bursátiles: rendimiento del mercado y de las acciones. 2013.

2. www.finanzas.com. Rendimiento de las acciones y los principales mercados bursátiles del mundo. 2013. 